\title{
Transmission Studies of Left-handed Materials
}

\author{
P. Markoš* and C. M. Soukoulis \\ Ames Laboratory and Department of Physics and Astronomy, Iowa State University, Ames, Iowa 50011
}

\begin{abstract}
Left-handed materials are studied numerically using an improved version of the transfer-matrix method. The transmission, reflection, the phase of the reflection and the absorption are calculated and compared with experiments for both single split-ring resonators (SRR) with negative permeability and left-handed materials (LHMs), which have both the permittivity $\epsilon$ and permeability $\mu$ negative. Our results suggest ways of positively identifying materials that have both $\epsilon$ and $\mu$ negative, from materials that have either $\mu$ or $\epsilon$ negative.
\end{abstract}

PACS numbers: 73.20.Mf, 41.20.Jb, 42.70.Qs

Photonic band gap (PBG) structures were originally introduced to control the electromagnetif wave propagation of matter国. Not only dielectric日e but metallic structure 3 - 5 were proposed for applications in the microwave and infrared regions. Very recently, a new area of research, called left-handed materials (LHM), has been introduced by Pendry et al $\mathbf{6}$. and Smith et al. $\mathrm{B}$. LHM are by definition composites, whose properties are not determined by the fundamental physical properties of their constituents but by the shape and distribution of specific patterns included in them. Thus, for certain patterns and distribution, the measured effective permittivity $\epsilon_{\text {eff }}$ and the effective permeability $\mu_{\text {eff }}$ can be made to be less than zero. In such materials, the phase and group velocity of an electromagnetic wave propagate in opposite directions giving rise to a number of novel properties?. This behavior has been called "left-handedness", a term first used by Veselago 10 over thirty years ago, to describe the fact that the electric field, magnetic intensity and propagation vector are related by a left-handed rule.

By combining a 2D array of split-ring resonators (SRRs) with a 2D array of wires, Smith et al. 1 demonstrated for the first timp the existence of left-handed materials. Pendry et al. has suggested that an array of SRRs give an effective $\mu_{\text {eff }}$, which can be negative close to its resonance frequency. It is also well known 3.6 that an array of metallic wires behaves like a high-pass filter, which means that the effective dielectric constant is negative at low frequencies. Very recently, Shelby et al.11 demonstrated experimentally that the index of refraction $n$ is negative for a LHM. Also, Pendry 22 has suggested that a LHM with negative $n$ can make a perfect lens.

\footnotetext{
* Permanent address: Institute of Physics, Slovak Academy of Sciences, Dúbravská cesta 9, 84228 Bratislava, Slovakia
}

In this Letter, we present systematic numerical results for the transmission, reflection and absorption properties of left-handed materials An improved version of the transfer-matrix method 13 (FMM) is used. Qualitative agreement with experiments 15 is obtained, and new predictions about the absorption and the phase of the reflection, to be checked experimentally, are presented.

The transfer-matrix method ${ }^{2} 3$ is used to calculate the EM transmission and reflection of LHMs. In the TMM, the total volume of the system is divided into small cells and fields in each cell are coupled to those in the neighboring cell. Then, the transfer matrix is defined by relating the incident fields on one side of the LHM structure with the outgoing fields on the other side. The main advantage of the TMM is the calculation of the transmission and reflection coefficients for EM waves of various frequencies incident on a finite thickness slab of a LHM. In this case, the material is assumed to be periodic in the directions parallel to the interfaces. The TMM has boen previously 1 applied in studies of metallic structures 354 , as well as in dielectric structures. In all these examples, the agreement between theoretical preflictions and experimental measurements was very good

Following the algorithm described in 13 , we develop the new version of the transfer-matrix code. The main change is the faster normalization of the transmitted waves in the calculation of the transmission coefficient through the structure. For the structures described in Figure 1, the typical CPU time necessary to calculate transmission for a given frequency is approximately 55 sec/unit cell on an alpha workstation 16 . Most of the CPU time is spent in the normalization of the actual numerical data. As our structure is highly non-homogeneous (permittivity of systems varies in three or more orders from site to site), we have to normalize the numerical data after 3-4 steps. For studies of more homogeneous samples, the CPU time could be reduced by a factor of 2 to 5 .

The TMM has been used to simulate the reflection and transmission from an array of square split-ring resonators (SRRs). Figure 1a shows a diagram of a single square SRR of the type used for our simulations and for experiment 15 . In our case we have $c=d=g=0.33$ $\mathrm{mm}$ and the size of SRR $w=3 \mathrm{~mm}$. Figure $1 \mathrm{~b}$ shows a three dimensional realization of the actual LHM that we have simulated.

Figure 2 presents the results of the transmission versus frequency for the split ring resonators alone, and of the LHM, which consists of the SRRs with metallic wires placed uniformly between the SRRs. The square array of metal wires alone behaves as a high pass filter with a cutoff frequency $\nu_{c}=19 \mathrm{GHz}$. The cutoff frequency $\nu_{c}$ 
of the metallic wires is given by $\nu_{c}=c_{\text {light }} / 2 d \sqrt{\epsilon_{0}}$, where $d$ is the distance between the wires, $c_{\text {light }}$ is the velocity of light in the air and $\epsilon_{0}$ is the dielectric constant of the background. In our case $d=5 \mathrm{~mm}$ and $\epsilon_{0}=1$. The cutoff frequency is independent of either value of $\operatorname{Re} \epsilon_{\mathrm{m}}$ or $\operatorname{Im} \epsilon_{\mathrm{m}}$ of the metal, provided that either $\left|\operatorname{Re} \epsilon_{\mathrm{m}}\right|>1000$ or $\operatorname{Im} \epsilon_{\mathrm{m}}>1000$. The dot-dashed curve is that of the SRR array with $d=5 \mathrm{~mm}$. By adding wires uniformly between the SRRs, a pass band occurs where both $\mu_{\text {eff }}$ and $\epsilon_{\text {eff }}$ are negative (solid line). The transmitted power of the LHM is very high (close to one), because no Im $\epsilon_{\mathrm{m}}$ is taken for the metal. We also studied more realistic cases with non-zero $\operatorname{Im} \epsilon_{\mathrm{m}}$. We found that the resonance frequency $\nu_{0}$ increases as $\left|\epsilon_{\mathrm{m}}\right|$ increases and saturates to a value of $\nu_{0} \approx 8.5$ as $\left|\epsilon_{\mathrm{m}}\right| \rightarrow \infty$. This is clearly shown in Fig. 3 where we plot $\nu_{0}$ versus the magnitude of $\epsilon_{\mathrm{m}}=$ $\epsilon_{r}+i \epsilon_{i}$. The resonance frequency is also sensitive to the permittivity of the board that the SRR is lying and of the embedding medium. We have found that $\nu_{0}$ drops as the dielectric constant of the embedding media $\epsilon_{a}$ increases. $\nu_{0}$ drops from its value of $8 \mathrm{GHz}$ for $\epsilon_{a}=1$ to a value of 6 $\mathrm{GHz}$ when $\epsilon_{a}=2.0$. The value $\nu_{0}$ increases by decreasing the value of the permittivity $\epsilon_{b}$ of the dielectric board. $\nu_{0}$ increases to a value of $9 \mathrm{GHz}$ from $8 \mathrm{GHz}$ when $\epsilon_{b}$ drops to a value of 1.4 from its original value of $\epsilon_{b}=3.4$.

The results presented in Fig. 2 are done by assuming that the permittivity of the metal $\epsilon_{\mathrm{m}}$ had a large negative value. As one can see from Fig. 2, the value of the transmission for the LHM is very close to one. This value drops considerable if one uses the fact that $\epsilon_{\mathrm{m}}$ for the metal has a complex permittivity. In Fig. 4a we present results similar to that in Fig. 2, with the only difference being the value of the $\operatorname{Im} \epsilon_{\mathrm{m}}$. Notice that the position of the resonance peak is at the same value at around 7.8 GHz. Due to the non-zero $\operatorname{Im} \epsilon_{\mathrm{m}}$ there is considerable drop of the peak of the transmission for the LHM. We have not done a systematic effort to fit the experimental results of Smith et al.

In Fig. 4b, we present new results of the frequency dependence of the absorption for either the SRR or the LHM. For the arrays of SRRs alone, we find that absorption has two peaks, shown as dashed lines in Fig. 4b, while the LHM has only one absorption peak (shown as a solid line in Fig. 4b) at the center of its resonance frequency. This might be a reasonable way of identifying materials that are left-handed and have both their $\epsilon_{\text {eff }}$ and $\mu_{\text {eff }}$ negative. On the inset of Fig. $4 \mathrm{~b}$ we present the frequency dependence of the efsorption as they were found for a simple model, using 6 : the following forms of the frequency dependence of the effective permittivity and permeability:

$$
\epsilon_{\mathrm{eff}}(\nu)=1-\frac{\nu_{p}^{2}}{\nu^{2}+i \nu \gamma}
$$

where $\nu_{p}$ is the plasma frequency or cut-off frequency $\nu_{c}$ of the wires, $\nu_{c}=\nu_{p} \approx 19 \mathrm{GHz}$ in our case. A negative

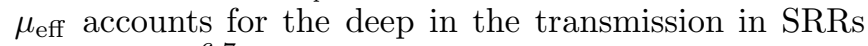
and its form 1 is given by

$$
\mu_{\mathrm{eff}}(\nu)=1-\frac{F \nu^{2}}{\nu^{2}-\nu_{0}^{2}+i \nu \Gamma}
$$

where $F$ is the filling factor which is in our case close to 0.3 and $\nu_{0} \approx 7.9 \mathrm{GHz}$ is the resonance frequency of the SRR. We used that $\gamma=\Gamma=0.2 \mathrm{GHz}$. There is very good agreement between the solution of present model and numerical simulations of real structures.

In Fig. 5 we present the results of the phase of the reflection for both the LHM and the array of SRRs. Notice that there is a substantial difference in their characteristics, which can help us identifying meta-materials that either have negative $\mu_{\text {eff }}$ or both negative $\mu_{\text {eff }}$ and $\epsilon_{\text {eff }}$.

Finally, in Fig. 6 we present results of our numerical experiments with various vacancies in SRR and LHM 7 . First, we remove one SRR from the structure. In PBG materials, where a gap is created by the interference effect of propagating waves, such a vacancy causes the appearance of sharp resonance frequency inside a gap. As it is seen in Fig 6a, the absence of one split ring resonator does not influences neither the position of the gap nor its form. Only the amplitude of the transmission decreases. This proves that the origin of the gap is not due to the interference effect but, as we assumed, in the negative effective permeability of the structure. Fig. 6 also shows how the transmission peak changes when one SRR or one wire is removed from the periodic structure of a LHM. In the absence of one SRR, the transmission peak decreases by more than one order of magnitude and becomes narrower (see Fig. 6a), while the wire vacancy creates an "inverse resonance" inside the transmission peak (see Fig. 6b).

We used an improved transfer-matrix method for numerical studies of complex meta-materials. Our numerical data of transmission confirms the presence of a resonant gap, in agreement with theoretical predictions and experiments. Our method enables us to analyze also the reflection and absorption of the light and also the change of the phase of the reflected field. Obtained numerical data are consistent with a simple homogeneous model defined by effective permittivity and permeability. This supports our belief that for the frequencies in the resonance gap our structure possesses negative effective $\mu_{\text {eff }}$ and negative effective $\epsilon_{\text {eff }}$. Although our method does not allow us to vary the size parameters of SRR continuously, we are able to predict, at least qualitatively, how the position of resonance gap depends on various parameters of the system. We believe that generalization of our numerical method to non-homogeneous discretization of space will enable us to analyze more realistic structures.

We thank D.R. Smith and I. El-Kady for fruitful discussions. Ames Laboratory is operated for the U.S.Department of Energy by Iowa State University under Contract No. W-7405-Eng-82. This work was supported by the Director of Energy Research, Office of Basic Science. P.M. thanks Ames Laboratory for its hospitality and support and Slovak Grant Agency for financial support. 
${ }^{1}$ Photonic Band Gap Materials, ed. By C. M. Soukoulis (Kluwer, Dordrecht), 1996.

2 J. D. Joannopoulos, R. D. Mead, and J. N. Winn, Photonic Crystal, (Princeton, 1995) and J. D. Joannopoulos, P. R. Villeneuve, and S. Fan, Nature 386, 143 (1997).

${ }^{3}$ D. R. Smith et al., Appl. Phys. Lett. 65, 645 (1994).

${ }^{4}$ E. Ozbay et al., Appl. Phys. Lett. 69, 3397 (1996); M. Sigalas et al., Phys. Rev. B 52, 11744 (1995).

${ }^{5}$ D. F. Sievenpiper et al., Phys. Rev. Lett. 80, 2829 (1998).

${ }^{6}$ J. B. Pendry et al., Phys. Rev. Lett. 76, 4773 (1996); J. Phys. Cond. Matter 10, 4785 (1998);

7 J. B. Pendry et al. IEEE Trans. Microwave Theory Tech. 47, 2075 (1999).

${ }^{8}$ D. R. Smith et al., Phys. Rev. Lett. 84, 4184 (2000); ibid. 85, 2933 (2000).

${ }^{9}$ Physics Today, May 2000 p. 17; J. B. Pendry, Phys. World 13, 27 (2000).

${ }^{10}$ V. G. Veselago, Sov. Phys. Usp. 10, 509 (1968).

${ }^{11}$ R. A. Shelby, D. R. Smith and S. Schultz, Science 292, 77 (2001).

12 J. B. Pendry, Phys. Rev. Lett. 85, 3966 (2000).

13 J. B. Pendry and A. MacKinnon, Phys. Rev. Lett. 69, 2772 (1992); J. B. Pendry, J. Mod. Opt. 41, 209 (1994).

${ }^{14}$ I. El-Kady et al., Phys. Rev. B 62, 15299 (2000).

${ }^{15}$ R. A. Shelby et al., Appl. Phys. Lett. 78, 489 (2001)

${ }^{16}$ A $533 \mathrm{MHz}$ Alpha 21164 processor with $512 \mathrm{MB}$ memory.

${ }^{17}$ In fact, due to the periodic boundary conditions in the $x$ and $y$ directions, we have a planar cavity inside the system.
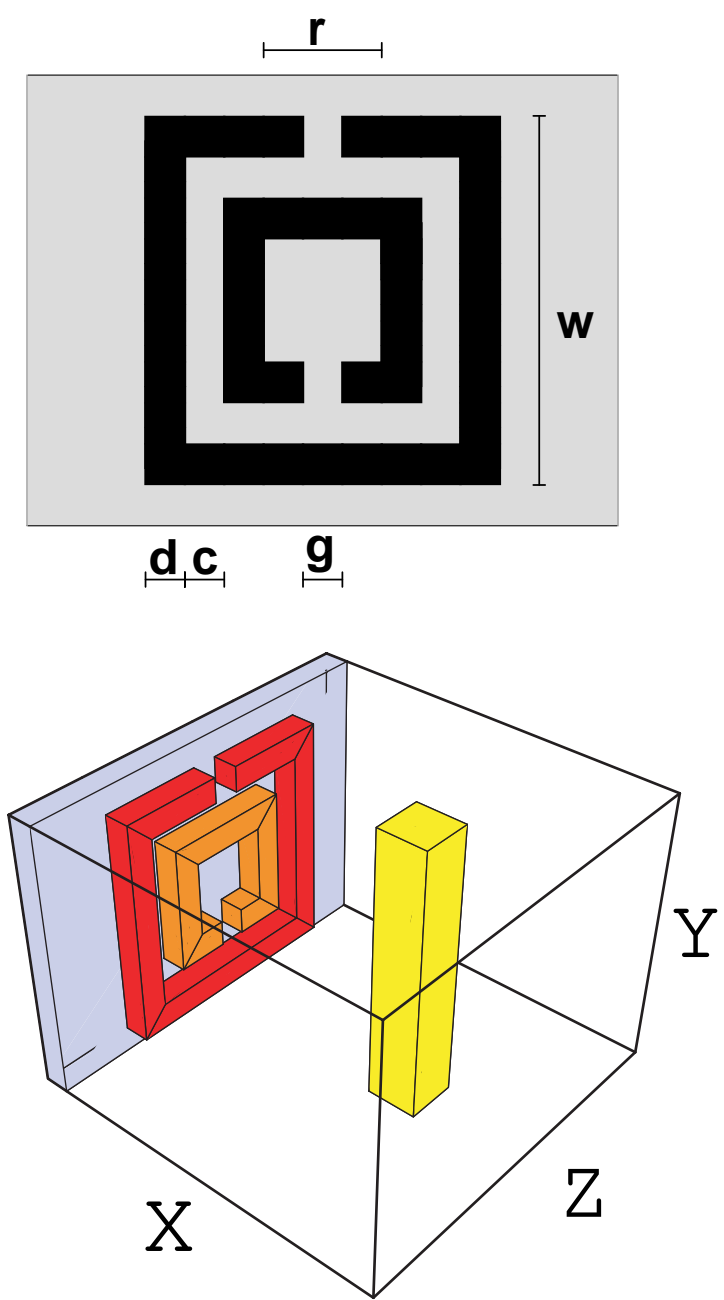

FIG. 1. (a) SRR consists from two splitted metallic rings located on a dielectric board. We approximate the rings by squares of size $w$. Other parameters, which influence the resonance frequency $\nu_{0}$ are the ring width $c$, the radial gap $d$, and the azimuthal gap $g$. (b) Configuration of the unit cell of a LHM. Light propagates along the $z$ axis and the magnetic intensity is parallel to the SRR axis, which is the $x$-axis. We assume periodic boundary conditions in $x$ and $y$ directions. In the numerical simulations the unit cell is $5 \times 3.63 \times 5 \mathrm{~mm}$ and is divided to $N_{x} \times N_{y} \times N_{z}=15 \times 11 \times 15$ small cells. This defines the length unit $=0.33 \mathrm{~mm}$. Number of unit cells in $z$ direction varies from 6 to 20 , and is 9 for most of the cases presented here. 


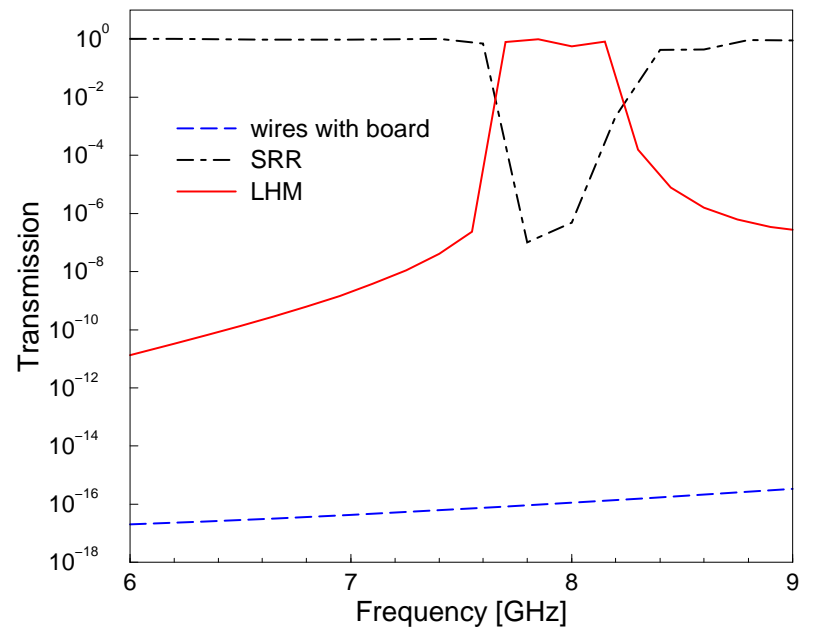

FIG. 2. Transmission of electromagnetic waves through arrays of SRRs, wires, and through a LHM with a real permittivity for the metal $\epsilon_{\mathrm{m}}=-3000$. The dielectric constant of the board is $\epsilon_{b}=3.4$. The thickness of wire is $1 \times 1 \mathrm{~mm}$. Magnetic intensity is parallel to the SRR axis. For the other polarization, no resonant gap was observed.

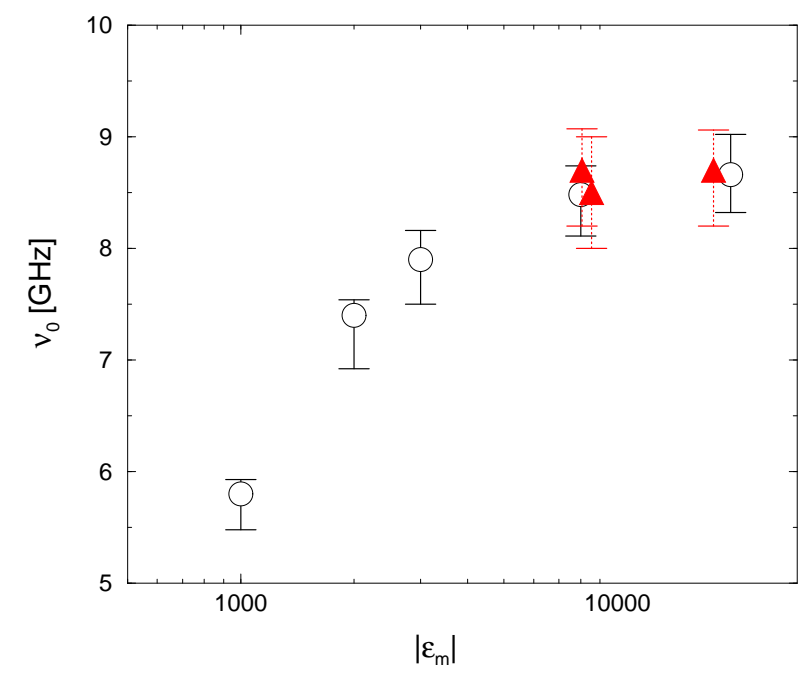

FIG. 3. Dependence of the resonance frequency $\nu_{0}$ on the absolute value of the permittivity $\left|\epsilon_{\mathrm{m}}\right|$ of metallic compounds. Both real (open circles) and complex (solid triangles) values of permittivity were considered. Error bars indicate the width of gap. $\nu_{0}$ does not depend on $\epsilon$ for $|\epsilon| \geq 10^{4}$.
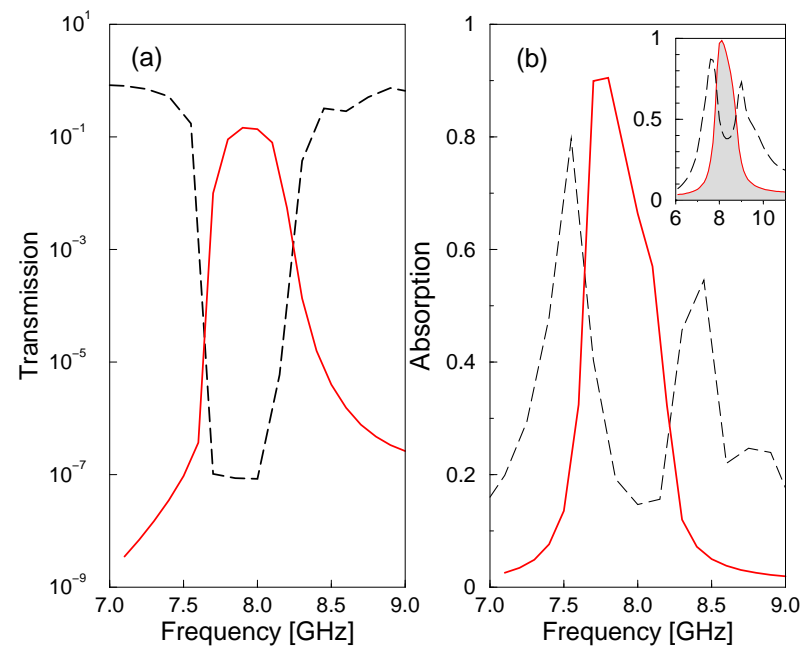

FIG. 4. (a) Transmission through arrays of SRRs (dashed line) and through a LHM (solid line) with a complex permittivity for the metal. (b) Absorption versus frequency for the same system as shown in (a). Permittivity of metal is $\epsilon=-3000+i 100$. Insert shows absorption in a composite system with a frequency dependent $\epsilon_{\mathrm{eff}}$ and $\mu_{\mathrm{eff}}$ given by Eqns. (11) and (2).

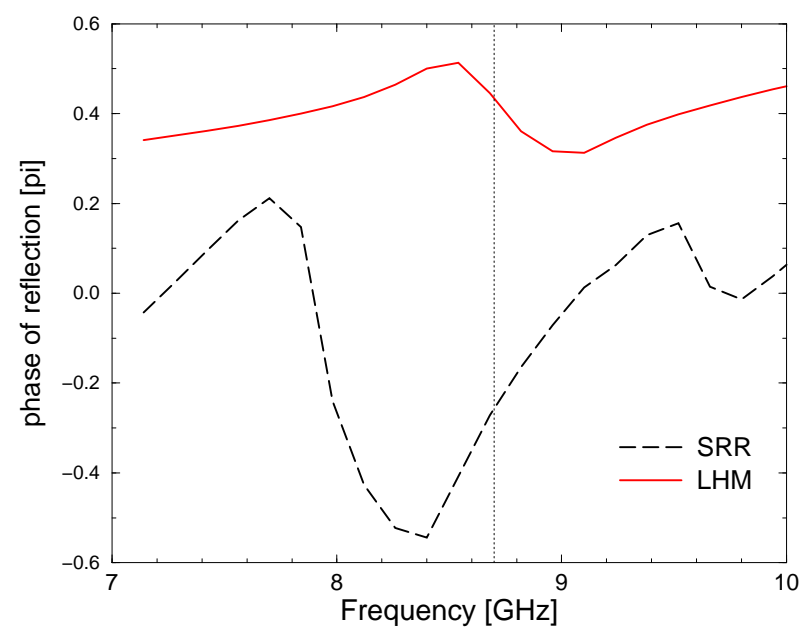

FIG. 5. Phase of reflection for both SRR and LHM. $\epsilon_{\mathrm{m}}=-1000+i 18000$. The vertical dotted line indicates the position of the center of the resonance gap $\nu_{0}$. 


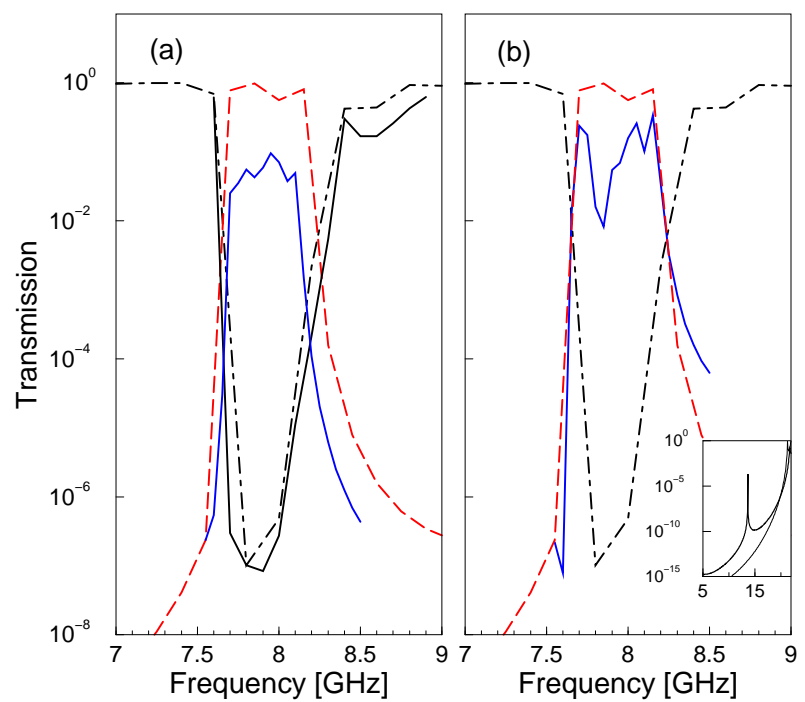

FIG. 6. Transmission of EM waves through SRRs (dot-dashed lines), and LHMs (dashed lines). In (a) one SSR has been removed and its transmission is shown by a solid line with no appreciable difference from the periodic case. In (b) one metallic wire has been removed and its transmission is shown as a solid line. In this case there is a sharp dip in the transmission of the LHM with an impurity. In all the cases $\epsilon_{m}=-3000$. For comparison, we also show as an inset in Fig. $6 \mathrm{~b}$ the transmission of waves through the lattice of wires only. A sharp resonance peak with a frequency $\nu_{\text {resonance }} \approx 13.65$ $\mathrm{GHz}$ appears when one wire is removed. Nine unit cells in the propagating direction are considered and the vacancies were created in the middle of the structure. 